Europhysics Letters

PREPRINT

\title{
Competition Between Exchange and Anisotropy in a Pyrochlore Ferromagnet
}

\author{
J. D. M. Champion ${ }^{1,2,3}$, S. T. Bramwell ${ }^{1}$, P. C. W. Holdsworth ${ }^{2}$ and M. J. \\ HARRIS $^{3}$ \\ 1 University College London, Department of Chemistry, 20 Gordon Street, London \\ WC1H0AJ, U.K. \\ 2 Laboratoire de Physique, Ecole Normale Supérieure, 46 Allée d'Italie, F69634 Lyon \\ cedex 07, France. \\ 3 ISIS Facility, Rutherford Appleton Laboratory, Chilton, Didcot, Oxon. OX11 OQX, \\ U.K.
}

PACS. 75.10.Hk - Classical spin models.

PACS. 75.40.Mg - Numerical simulation studies.

PACS. 75.30.Kz - Magnetic phase boundaries.

\begin{abstract}
The Ising-like spin ice model, with a macroscopically degenerate ground state, has been shown to be approximated by several real materials. Here we investigate a model related to spin ice, in which the Ising spins are replaced by classical Heisenberg spins. These populate a cubic pyrochlore lattice and are coupled to nearest neighbours by a ferromagnetic exchange term $J$ and to the local $\langle 1,1,1\rangle$ axes by a single-ion anisotropy term $D$. The near neighbour spin ice model corresponds to the case $D / J \rightarrow \infty$. For finite $D / J$ we find that the macroscopic degeneracy of spin ice is broken and the ground state is magnetically ordered into a four-sublattice structure. The transition to this state is first-order for $D / J>5$ and secondorder for $D / J<5$ with the two regions separated by a tricritical point. We investigate the magnetic phase diagram with an applied field along $[1,0,0]$ and show that it can be considered analogous to that of a ferroelectric.
\end{abstract}

Background. - Geometrical frustration represents a recipe by which condensed matter can be disordered even in the absence of substitutional disorder [1]. The canonical example is the proton disorder in ice, which was famously shown by Pauling to be the origin of the experimentally observed ground state entropy [2, 3]. Anderson later illustrated a direct mapping of Pauling's model onto the Ising antiferromagnet on the pyrochlore lattice [- 4 . However, Anderson's antiferromagnet does not appear to occur in nature and the mapping was for many years something of an academic curiosity, albeit one that inspired interest in frustrated magnetism $[5]$. Recently, however, this has changed with the presentation of an alternative mapping named "spin ice", motivated by experiments on the cubic pyrochlore $\mathrm{Ho}_{2} \mathrm{Ti}_{2} \mathrm{O}_{7}$ [8] . This material is closely approximated by a pyrochlore ferromagnet with $\langle 1,1,1\rangle$ Ising-like spins, which maps onto the ice model [8] and hence onto Anderson's antiferromagnet [9, 10]. The behaviour of $\mathrm{Ho}_{2} \mathrm{Ti}_{2} \mathrm{O}_{7}$ [8] and the related compound $\mathrm{Dy}_{2} \mathrm{Ti}_{2} \mathrm{O}_{7}$ [13, 14] is described to

(C) EDP Sciences 

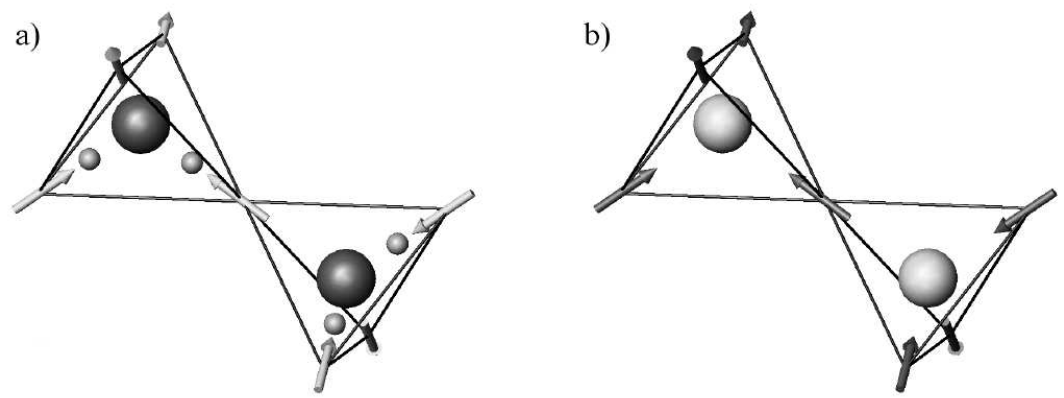

Fig. 1 - The spin ice mapping [8,9]. a) Coordination of $\mathrm{H}_{2} \mathrm{O}$ molecules in ice represented by proton displacement vectors. b) Spins in the spin ice model.

a good first approximation by the spin ice model and has recently been shown to be reproduced to an excellent degree by a related "dipolar spin ice model" including long range dipole interactions [15, 16, 18]. These materials are part of a series of rare earth titanates that are in general of interest for their frustrated magnetic behaviour [11, 12].

The mapping may be illustrated as follows [8] (see Fig. 11). In $\mathrm{Ho}_{2} \mathrm{Ti}_{2} \mathrm{O}_{7}$ and $\mathrm{Dy}_{2} \mathrm{Ti}_{2} \mathrm{O}_{7}$ the magnetic rare earth ions occupy a cubic pyrochlore lattice, an array of corner-linked tetrahedra. This lattice is the medial lattice (i.e. the lattice formed by the mid-points of the bonds) of the diamond-type oxide sublattice in cubic ice 任. The proton disorder of ice can be described by displacement vectors that populate the vertices of this lattice. The "ice rules", the condition that there are two protons near to, and two further away from each oxide, corresponds to the condition that two vectors point into and two point out of each tetrahedron. In $\mathrm{Ho}_{2} \mathrm{Ti}_{2} \mathrm{O}_{7}$ and $\mathrm{Dy}_{2} \mathrm{Ti}_{2} \mathrm{O}_{7}$ the rare earth ground state is an effective $\mathrm{S}=1 / 2$ doublet with local $\langle 1,1,1\rangle$ quantization axis and so can be represented by a classical Ising-like vector analogous to a proton displacement vector. Net ferromagnetic coupling between near neighbour spins completes the analogy with the ice model, corresponding to the frustrated proton-proton repulsion imposed by the ice structure. The spin ice model describes qualitatively the results of many experiments on these materials, including the absence of magnetic order down to the lowest temperatures [8, 11, 14].

The Model. - The macroscopic degeneracy of the spin ice model makes it very susceptible to perturbations. That is, one can expect correction terms to the idealized Hamiltonian to dramatically change the low temperature properties by lifting the degeneracy, moving the system into a long range ordered state. This is the case for dipolar interactions, as long as thermodynamic equilibrium is maintained. In this paper we pursue the effects of perturbation by studying a continuous spin ice model, with classical Heisenberg spins replacing the discrete Ising-like degrees of freedom. Our model is defined by the spin Hamiltonian

$$
H=-J \sum_{<i, j>} \vec{S}_{i} \cdot \vec{S}_{j}-D \sum_{i}\left(\vec{S}_{i} \cdot \vec{d}_{i}\right)^{2}-\sum_{i} \vec{H} \cdot \vec{S}_{i}
$$

where the $\vec{S}_{i}$ are classical vectors of unit length. The cubic crystal fields of magnitude $D$ are along the four $\langle 1,1,1\rangle$ directions for the four spins of the primitive unit cell. The extra refinement of continuous, rather than Ising spins would be expected to capture the effect of quantum fluctuations, which must be present in some extent in all real materials. One would also expect it to be an accurate starting Hamiltonian for any prospective ice or spin 
a)

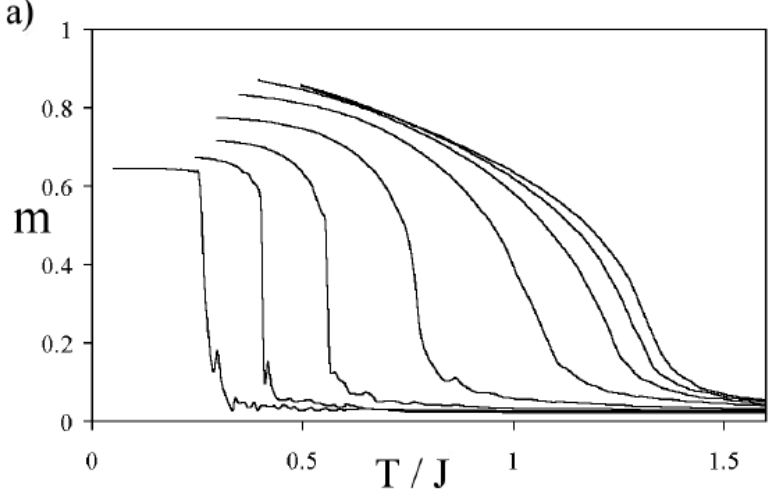

b)

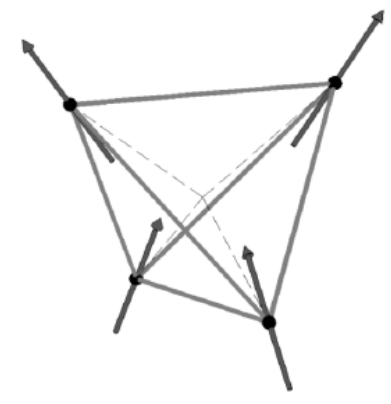

Fig. 2 - a) Monte Carlo simulation of the magnetization versus temperature for Hamiltonian 1 . Thermal hysteresis was observed for large $D / J$; the results shown are cooling curves at (from left to right) $D / J=22.91,15.49,10.47,7.08,4.79,3.24,2.18,1.0$. b) Spin structure of the $q=0$ basis for $D / J=7$, illustrating the canting of the spins towards $[0,0,1]$.

direction.

ice like materials with an exchange constant $J$ in excess of the $O\left(1^{\circ}\right) K$ temperature range of $\mathrm{Ho}_{2} \mathrm{Ti}_{2} \mathrm{O}_{7}$ and $\mathrm{Dy}_{2} \mathrm{Ti}_{2} \mathrm{O}_{7}$, where dipolar interactions are important. The Hamiltonian (ip) describes the transition from spin ice $(D / J \rightarrow \infty)$ to ordinary ferromagnetic $(D / J \rightarrow 0)$ behaviour. We have investigated it by Monte Carlo simulation using the standard Metropolis spin-flipping algorithm for systems of size $N=432-11634$ spins. All simulation lengths were 100,000 Monte Carlo steps per spin with 30-40,000 steps used for initial equilibration.

Spontaneous Magnetization. - Our first result is illustrated in Fig. 2, where we plot Monte Carlo simulation results of the scalar magnetization per spin $m=\frac{1}{N}\left\langle\sqrt{\sum_{i}\left(\vec{S}_{i}\right)^{2}}\right\rangle$ as a function of temperature $T / J$ for $0<D / J<25$. For all the finite $D / J$ investigated there is a transition to an ordered state with $q=[0,0,0]$ propagation vector. It is clear that the transition only disappears in the spin ice limit, $D / J \rightarrow \infty$ and therefore that the infinite ground state degeneracy is lifted, for all finite $D / J$, in favour of a long range ordered $q=0$ state. The transition is marked by a maximum in specific heat and susceptibility. It is second-order for $D=0$ (the simple ferromagnetic case) but clearly first-order for large $D / J$, suggesting the presence of a tricritical point [19,20] at intermediate $D / J$. To locate the tricritical point $D_{t c} / J$ we have performed a finite size scaling analysis of the maximum in the susceptibility $\chi_{\max } \cdot \chi_{\max }$ should scale as $L^{\gamma / \nu}$ in the second-order regime [21], where $\gamma$ and $\nu$ are the critical exponents describing the divergence of the susceptibility and correlation length. Assuming the Ising universality class, one has $\gamma / \nu=1.969 \approx 2$ [22]. Such $L^{2}$ scaling was observed for $D / J<5$ only, and a careful analysis suggests that the tricritical point occurs at $D_{t c} / J=5.0 \pm 0.5$.

To further understand the results of Fig. 2, consider the nature of the ordered state: a $q=0$ state has a magnetic unit cell consisting of a single tetrahedron. For the Ising system $(D / J \rightarrow \infty)$ the spins are directed along the local $\langle 1,1,1\rangle$ axes, as shown in Fig. 1 b b. We call this the " $\langle 1,1,1\rangle$ " state which has energy per spin $E_{\langle 1,1,1\rangle}=-J / 3-D$ and overall magnetization $m=1 / \sqrt{3}$ along $\vec{z}=[0,0,1]$. The state is frustrated by the crystal field with, for each tetrahedron, four bonds making a contribution of $-J / 3$ and two making a 
contribution of $J / 3$. Introduction of continuous spins allows the system to regain frustrated bond energy at the expense of field energy by relaxing away from the body centered cubic directions; see Fig. 2b. In disordered spin ice ground states this relaxation is itself frustrated and it is easy to see that the maximum energy gain will occur for relaxation away from the ordered $q=0$ state. For finite $D / J$ we therefore expect degeneracy to be lifted in favour of this state and to have a canting of the spins towards the $[0,0,1]$. In the limit $D / J \rightarrow 0$ one should obtain a perfectly aligned ferromagnetic (FM) state, which we call the "[0, 0,1$]$ state". For finite $D$ it has energy $E_{[0,0,1]}=-3 J-D / 3$.

We have estimated the ground state spin orientation for large and small crystal field by making the reasonable ansatz that the relaxation away from, or towards the $\langle 1,1,1\rangle$ is homogeneous for the four spins of the unit cell. Comparing with numerical data this seems $a$ posteriori to be correct. For small $\alpha=D / J$ a small relaxation away from $[0,0,1]$ and towards $\langle 1,1,1\rangle$ gives for the magnetization $m$ and the energy $E$ per spin

$$
E=J\left(-3-\frac{\alpha}{3}-\frac{\alpha^{2}}{18}\right), m=\sqrt{\left(1-\frac{\alpha^{2}}{72}\right)} .
$$

This result illustrates the fact that the energy and magnetization change very slowly from the ferromagnetic value for $D$ increasing from zero to $D \sim O(J)$. In the opposite limit of strong crystal field, we define $\gamma=J / D$ and allow a small relaxation away from the $\langle 1,1,1\rangle$ state towards the $[0,0,1]$ state. In this case we find for small $\gamma$

$$
E=D\left(-1-\frac{\gamma}{3}-\frac{32 \gamma^{2}}{9}\right), \quad m=\frac{1}{\sqrt{3}}+\frac{8 \gamma}{3 \sqrt{3}}
$$

which again suggests a slow change in magnetization with $D / J$.

To test these expressions, the magnetization was computed by performing zero temperature Monte Carlo simulations. In Fig. 3 we plot these estimates versus the anisotropy $D / J$ and compare with the small anisotropy result (2) and the large anisotropy result (3). The agreement is seen to be excellent in both cases, with each asymptotic equation breaking down near the tricritical point at $D_{t c} / J=5$. It is therefore tempting to associate the change in order of the transition with a non-linear cross over from a regime that is nearly ferromagnetic, to one that is nearly $q=0$ spin ice.

Magnetic Phase Diagram. - Further insight into the nature of this cross over is gained by a consideration of the magnetic phase diagram. We consider the behaviour of the continuous spin ice model in an external magnetic field applied along the $[1,0,0]$ direction which sustains the symmetry of the ordered state for all finite $D / J$. In ref.( [13]) we showed that the magnetic phase diagram of the near neighbour spin ice model with the field along $[1,0,0]$ has two lines of first-order phase transitions that separate phases of different magnetization. Both lines terminate in critical points, and we have argued that the phase diagram can be considered analagous to that of a liquid-gas system, where a line of first-order transitions separates phases of different density. For the continuous spin ice model we have mapped out the equivalent phase diagram by Monte Carlo simulation. In each case the phase boundary was estimated from the maximum in the magnetic susceptibility. In principle, now we know the true ground state, we could measure the true order parameter and its fluctuations, by calculating the projection onto the calculated state.

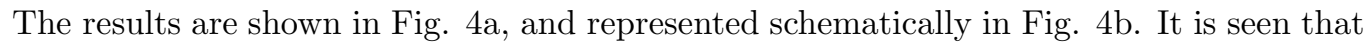
the two first-order lines of the spin ice phase diagram coalesce below the ordering temperature,

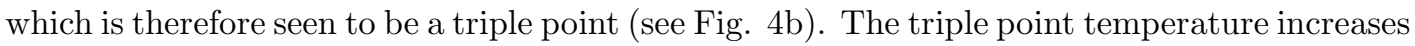




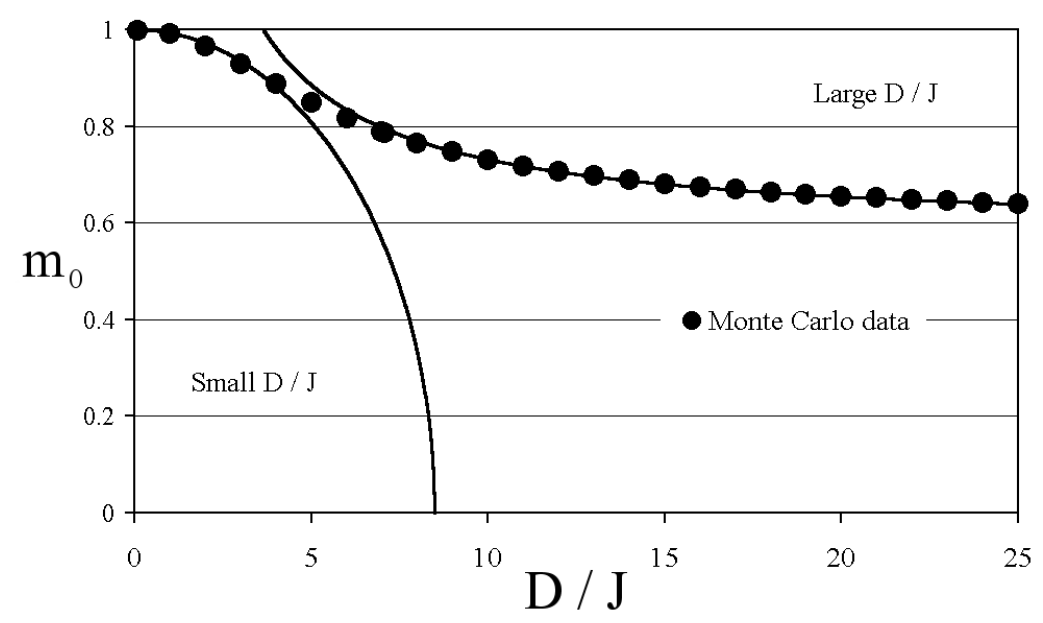

Fig. 3 - Comparison of the numerical zero temperature magnetization $m_{0}$, estimated by a zerotemperature Monte Carlo method, with that estimated by the analytical expressions (2) (left hand line) and (3) (right hand line). Both asymptotic expressions are seen to break down near the tricritical point $D_{t c} / J=5.0 \pm 0.5$.

with decreasing $D / J$ and eventually the two "wings" disappear and give way to a line of firstorder transitions along the zero field axis, typical of a ferromagnet. We would anticipate that the two wings disappear precisely at the tricritical point determined above, $D_{t c} / J \approx 5$. This kind of phase diagram is exhibited by idealised ferroelectrics and also some real ones such as $\mathrm{BaTiO}_{3}$ [23]. It can be rationalised by Landau theory [23, 24], where the free energy is written $G=G_{0}+\frac{c_{2}}{2} m^{2}+\frac{c_{4}}{4} m^{4}+\frac{c_{6}}{6} m^{6}-N m H$. Here, $m$ and $H$ are the order parameter and conjugate applied field and the $c_{n}$ 's are constants related to the n-th order susceptibilities. If $c_{4}>0$ then the zero-field transition is second-order and if $c_{4}<0$ it is first-order, with the characteristic "winged" phase diagram. The slopes of the wings are approximately $\sim \sqrt{\frac{c_{4}}{c_{2}}}$. In general, termination points of first-order lines need not be associated with critical fluctuations, in which case they should be termed "first-order" critical points. One such point is known to occur on the zero-field axis of the phase diagram of the one dimensional Ising ferromagnet with additional inverse square interaction [26]. In fact it has been suggested 24 that the end-point of a line of symmetry sustaining transitions is usually first-order, the liquid gas transition being a special case. In general, at a first-order critical point, the susceptibility will diverge, but there may or may not be critical fluctuations [24,25]). A detailed analysis of this question with regard to the current system would be an interesting topic of future study.

Conclusions. - In conclusion, it is noteworthy that the continuous spin ice model displays behaviour characteristic of ferroelectrics. It is plausible that ice itself would exhibit such a phase diagram if the dynamics did not become immeasurably slow at temperatures well above the triple point [3]. However ice is not proton ordered in the absence of defects or stabilizing surfaces and there is much debate concerning the true nature of its ground state 27 31. Very similar remarks apply to $\mathrm{Ho}_{2} \mathrm{Ti}_{2} \mathrm{O}_{7}$, where the dynamics also become immeasurably slow below $0.7 \mathrm{~K}$. In view of our results, it might be that the ordered state favoured by the long range part of the dipolar coupling competes with that favoured by continuous spins. 
a)

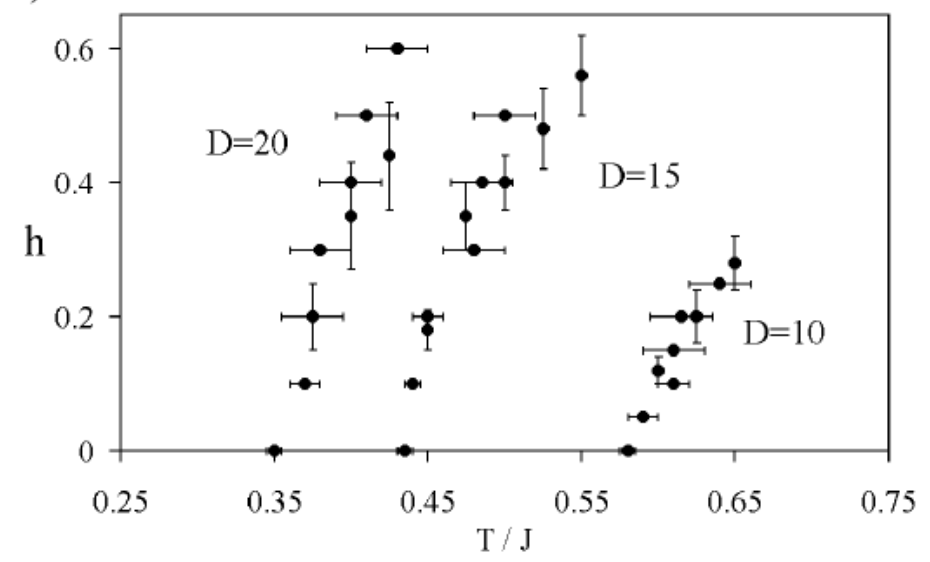

b)

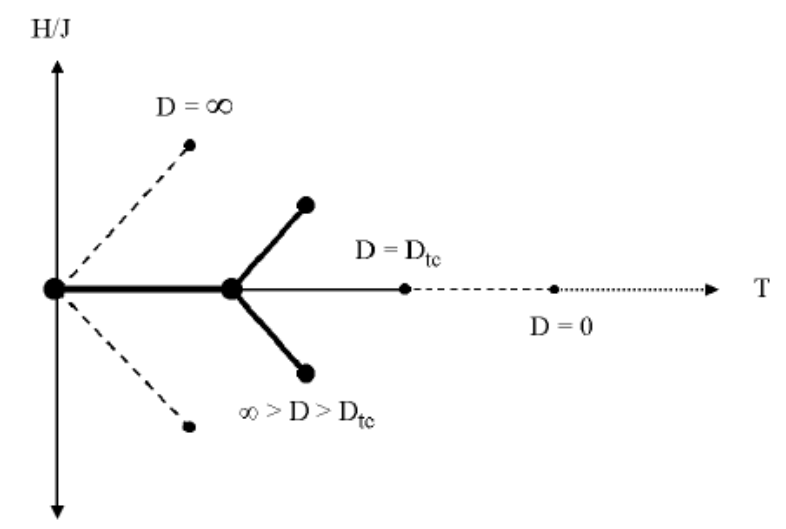

Fig. 4 - Magnetic phase diagram for the continuous spin ice model define by eqn. (1). (a) Phase diagram determined by Monte Carlo simulation on a system of $N=2000$ spins. Points on the coexistence lines were determined from the maximum in the susceptibility in fixed temperature (vertical error bars) or fixed field (horizontal error bars) Monte Carlo runs. For ease of comparison with experiment and ref.( [13]), the field $h$ is defined as $h=H \times\left(\mu_{\mathrm{B}} / k_{\mathrm{B}}=H / 0.06717\right.$, with $\mu_{\mathrm{B}}$ and $k_{\mathrm{B}}$ the Bohr magneton and Boltzmann's constant respectively, and $H$ defined in eqn.1. (b) Schematic phase diagram showing the case of $D / J=0$ (short dashed line), $D / J=D_{t c} / J$ (thin line), $D / J>D_{t c} / J$ (thick line) and $D / J \rightarrow \infty$ (long dashed line)

Through the application of a magnetic field in the $[1,1,0]$ direction [8], it is possible to put $\mathrm{Ho}_{2} \mathrm{Ti}_{2} \mathrm{O}_{7}$ into an ordered state of the same symmetry as the ground state of the dipolar spin ice model [16]. It would be interesting to prepare a sample in this way at a temperature below the expected ordering transition at $0.18 \mathrm{~K}$, and to examine the stability of the ordered state as the field is removed. At a theoretical level, it would be extremely interesting to consider the effect of continuous spins on the dipolar spin ice model of den Hertog and Gingras [15], 
that is an accurate description of $\mathrm{Ho}_{2} \mathrm{Ti}_{2} \mathrm{O}_{7}$ and $\mathrm{Dy}_{2} \mathrm{Ti}_{2} \mathrm{O}_{7}[15,18]$.

$* * *$

It is a pleasure to thank J.T. Chalker and M.J.P. Gingras for stimulating discussions. JDMC thanks the ENS, the EPSRC and ISIS for financial support. This work was supported by the Pôle Scientifique de Modélisation Numérique at the École Normale Supérieure de Lyon.

\section{REFERENCES}

[1] Ziman J.M., Models of disorder (C.U.P.) 1979

[2] Pauling L., J. Am. Chem. Soc., 57 (2680) 1935.

[3] Giauque W. F. and Stout J. W., J. Am. Chem. Soc., 58 (1144) 1936.

[4] Anderson P. W., Phys. Rev., 102 (1008) 1956.

[5] Greedan J. E., J. Mater. Chem. 11 (37) 2001.

[6] Moessner R., cond-mat/0010301

[7] Shender E.F. And Holdsworth P. C. W., Fluctuations and Order: A New Synthesis, edited by Millonas M. M . (Springer-Verlag) 1996

[8] Harris M. J., Bramwell S. T., McMorrow D. F., Zeiske T. , Godfrey K. W. , Phys. Rev. Lett., 79 (2554) 1997.

[9] Bramwell S. T. and Harris M. J., J. Phys-Condens. Mat., 10 ( L215) 1998.

[10] Moessner R. , Phys. Rev. B, 57 (R5587) 1998.

[11] Harris M. J. et al., J. Magn. Mag. Mat., 177-181 (757) 1998.

[12] Gingras M. J. P. et al, Phys. Rev. B, 62 (6496) 2000.

[13] Harris M. J. et al., Phys. Rev. Lett., 81 (4496) 1998.

[14] Ramirez A. P. et al., Nature, 399 (333) 1999.

[15] Den Hertog B. C. And Gingras M. J. P., Phys. Rev. Lett., 84 (3430) 2000.

[16] den Hertog B. C., Melko R. G. and Gingras M. J. P., cond-mat/0009225

[17] den Hertog B. C. and Gingras M. J. P., cond-mat/0012275.

[18] Bramwell S. T. et al., cond-mat/0101114

[19] Griffiths R. J., Phys. Rev. B, 7 (545) 1973.

[20] Herrmann H. J. and Landau D. P., Phys. Rev. B, 48 (239) 1993.

[21] Cardy J. L., Scaling and Renormalization Group (Cambridge University Press) 1996

[22] Binney J. J., And Dowrick N. J. and Fisher A. J. And Newman M. E. J., The Theory of Critical Phenomena (Oxford University Press) 1992

[23] Mitsui T., Tatsuzaki I. and Nakamura E., An Introduction to the Physics of Ferroelectrics, Ferroelectricity and Related Phenomena, Vol. 1, edited by LeFkowitz I. AND TAYlor G. I. ( Gordon and Breach Science Publishers, London ) 1986

[24] Aharony A., Critical Phenomena, Lecture Notes in Physics, Vol. 186, edited by Hahne F. (Springer-Verlag, Berlin Heidelberg) 1983, p. 209.

[25] Fisher M. E. And Berker A. N., Phys. Rev. B.., 26 (2507) 1982.

[26] Thouless D. J., Phys. Rev. B., 187 (732) 1969.

[27] Barkema G.T. and Deboer J, J. Chem. Phys., 99 (2059) 1993.

[28] Davidson E. R. And Morokuma K., J. Chem. Phys., 81 (3741) 1984.

[29] Jackson, S. M. et al, J. Phys. Chem. B , 101 (6142) 1997.

[30] Lekner J., Physica B, 252 (149) 1998.

[31] Buch V., Sandler P. And Sadlej J., J. Phys. Chem. B, 102 (8641) 1998. 